\title{
Phenotypic and Molecular Characterization of Commensal, Community-Acquired and Nosocomial Klebsiella spp.
}

\author{
Marta Gómez $^{1}{ }^{\mathbb{D}}$, Arancha Valverde ${ }^{2}$, Rosa del Campo ${ }^{2}$, Juan Miguel Rodríguez ${ }^{1}(\mathbb{D}$ and \\ Antonio Maldonado-Barragán 1,3,*(D) \\ 1 Department of Nutrition and Food Science, Complutense University of Madrid, 28040 Madrid, Spain; \\ marta_gmz@hotmail.com (M.G.); jmrodrig@ucm.es (J.M.R.) \\ 2 Department of Microbiology, Hospital Universitario Ramón y Cajal IRYCIS, 28034 Madrid, Spain; \\ aranchavalverde@gmail.com (A.V.); rosa.campo@salud.madrid.org (R.d.C.) \\ 3 Infection and Global Health Research Division, School of Medicine, University of St. Andrews, North Haugh, \\ St Andrews KY16 9TF, UK \\ * Correspondence: amb52@st-andrews.ac.uk
}

\section{check for} updates

Citation: Gómez, M.; Valverde, A.; del Campo, R.; Rodríguez, J.M.; Maldonado-Barragán, A. Phenotypic and Molecular Characterization of Commensal, Community-Acquired and Nosocomial Klebsiella spp.Microorganisms 2021, 9, 2344. https://doi.org/10.3390/ microorganisms 9112344

Academic Editor:

Dobroslava Bujňáková

Received: 19 October 2021

Accepted: 9 November 2021

Published: 12 November 2021

Publisher's Note: MDPI stays neutral with regard to jurisdictional claims in published maps and institutional affiliations.

Copyright: (c) 2021 by the authors. Licensee MDPI, Basel, Switzerland. This article is an open access article distributed under the terms and conditions of the Creative Commons Attribution (CC BY) license (https:// creativecommons.org/licenses/by/ $4.0 /)$.

\begin{abstract}
Klebsiella spp. is a relevant pathogen that can present acquired resistance to almost all available antibiotics, thus representing a serious threat for public health. While most studies have been focused on isolates causing community-acquired and nosocomial infections, little is known about the commensal isolates colonizing healthy subjects. We describe the molecular identification and the phenotypic characterization of commensal Klebsiella spp. from breast milk of healthy women and faeces from healthy breast-fed infants, which were compared with isolates from community-acquired infections and from a nosocomial NICU outbreak. The phylogenetic analysis of a 454-bp sequence of the rpoB gene was useful for species identification (K. pneumoniae, K. variicola, K. quasipneumoniae, K. oxytoca, K. grimontii, K. michiganensis, Raoultella planticola and R. ornithinolytica), previously misidentified as K. pneumoniae or K. oxytoca by biochemical methods. Globally, we report that commensal strains present virulence traits (virulence genes, siderophores and biofilms) comparable to community-acquired and NICU-infective isolates, thus suggesting that the human microbiota could constitute a reservoir for infection. Isolates causing NICU outbreak were multi-drug resistant (MDR) and ESBLs producers, although an imipenem-resistant commensal MDR K. quasipneumoniae isolate was also found. A commensal K. pneumoniae strain showed a potent bacteriocin-like inhibitory activity against MDR Klebsiella isolates, thus highlighting the potential role of commensal Klebsiella spp. in health and disease.
\end{abstract}

Keywords: Klebsiella; rpoB; virulence; siderophores; biofilms; antibiotic resistance; bacteriocins

\section{Introduction}

Klebsiella spp. are ubiquitous in nature and can be found in environment samples (surface water, sewage, soil and plants) and, also, colonizing the mucosal surfaces of healthy mammals [1]. The major species of this genus is Klebsiella pneumoniae, followed by Klebsiella oxytoca, both considered as opportunistic pathogens with major relevance in community- and hospital-acquired (nosocomial) infections, which are particularly severe in immunocompromised subjects such as those hospitalized in transplant, intensive care (ICU), or neonatal units (NICU) [2].

Phylogenetic analyses have shown that K. pneumoniae complex comprises seven phylogenetic groups (Kp1 to Kp7). Kp1 is the most abundant and includes K. pneumoniae sensu stricto; Kp2, Kp3, Kp4, Kp5, Kp6 and Kp7 include K. quasipneumoniae subsp. quasipneumoniae, K. variicola subsp. variicola, K. quasipneumoniae subsp. similipneumoniae, K. variicola subsp. tropicalensis, K. quasivariicola and K. africanensis, respectively [3-5]. On the other hand, the K. oxytoca complex comprises six phylogroups, Ko1, Ko2, Ko3, Ko4, Ko6 and Ko8, 
including K. michiganensis, K. oxytoca sensu stricto, K. spallanzanii, K. pasteurii, K. grimonti and $K$. huaxiensis $[4,6]$.

Isolates grouped in the K. pneumonie complex possess similar biochemical and phenotypic features, being inaccurately identified as K. pneumoniae sensu stricto or as K. variicola by conventional microbiological methods. Thus, a recent study has shown that $K$. variicola and K. quasipneumoniae, which are often misidentified as K. pneumoniae, cause severe lifethreatening infections similar to K. pneumoniae [7]. In the same manner, the members of the K. oxytoca complex are frequently misidentified as K. oxytoca sensu stricto. However, it has been suggested that $K$. michiganensis, which is commonly erroneously identified as K. oxytoca, is likely to be more clinically relevant than K. oxytoca in human-associated infections [8].

Commensal colonizing isolates have been far less studied than clinically relevant community-acquired and nosocomial Klebsiella spp. causing infectious diseases. In fact, studies about commensal Klebsiella isolates from healthy non-hospitalized subjects are currently very scarce. By using culture-independent methods, it has been estimated that approximately $3.8 \%$ of stool samples from healthy individuals contains K. pneumoniae [9]. In a recent study with healthy adults, it has been shown that although Klebsiella spp. constitute minor bacterial components of the human gut microbiota, some K. pneumoniae isolates could present a great potential to cause infections [10]. This was previously observed in a study with healthy Korean adults, where a high proportion of subjects showed faecal carriage of K. pneumoniae sequence type 23, which is associated with pyogenic liver abscess in Korea [11]. In fact, two recent studies with hospitalized adults have shown that gastrointestinal colonization with K. pneumoniae is strongly linked to subsequent infections in these subjects during hospitalization and demonstrated that a large proportion of $K$. pneumoniae infections were acquired from patient's own microbiota [12,13].

Nosocomial Klebsiella infections are especially problematic in preterm neonates causing neonatal sepsis, including both early- and late-onset infections [14]. In a recent work, it has been shown that "healthy" antibiotic-treated preterm infants hospitalized in NICUs can harbour different Klebsiella spp. such as K. pneumoniae, K. quasipneumoniae, K. grimontii and K. michiganensis, which could greatly contribute to the resistome [8].

Overall, all these studies suggest that Klebsiella spp. is a habitual commensal in the healthy human microbiota, which could provide a potential reservoir for infection. In the light of these findings, the phenotypic and molecular characterization of Klebsiella isolates from healthy subjects could contribute to understanding the relevance of commensal Klebsiella spp. as a reservoir of potentially dangerous traits for human health. Previous works have determined that this genus can be a part of the human milk microbiota of healthy women [15], frequently arising from the use of pumps for milk expression [16]. Breastfeeding women are a representative sample of the general population since the majority of them will give birth at some point in a hospital, where the newborn will be ideally breastfeed within the first hour of birth. As a consequence, human milk represents one of the first vehicles for the mother-to-infant transfer of microbes [17].

In these studies, we obtained a collection of Klebsiella isolates from the milk of healthy women and from the meconium and faeces of breast-fed term infants. The aim of this study was to identify and characterize these commensal Klebsiella isolates and compare them with nosocomial isolates from an NICU outbreak and from community-acquired infections isolates.

\section{Materials and Methods}

\subsection{Bacterial Strains and Growth Conditions}

A total of 56 Klebsiella spp. isolates, which were initially identified as K. pneumoniae $(n=35)$ or K. oxytoca $(n=21)$ by routine biochemical methods (Wider system; Francisco Soria Melguizo, S.A., Madrid, Spain), were included in this study (Table 1). These isolates were obtained from different origins: (i) 20 commensal isolates from human milk $(n=5)$, meconium $(n=1)$ and faeces of breast-fed infants $(n=14)$ of healthy individuals from 
the bacterial collection of the research group 920080 (Complutense University of Madrid, Spain); (ii) 26 community-acquired isolates causing bacteraemia in adult outpatients at the Hospital Ramón y Cajal (Madrid, Spain); and (iii) 10 clinical isolates from the neonatal ICU of the Hospital 12 de Octubre (Madrid, Spain) from blood $(n=2)$, catheter $(n=1)$, environmental surfaces $(n=2)$ and colonizing the gut of newborns admitted at NICU $(n=5)$. The Ethical Committee on Clinical Research of the Hospital Clínico San Carlos of Madrid (Spain) approved the study protocol (reference 10/017-E). In the frame of such protocol, samples used to isolate the bacterial strains were obtained after informed written consent of each person or, when required, of the infants' legal guardians. All strains were routinely grown in Brain Heart Infusion (BHI; Oxoid, Basingstoke, UK) broth, BHI solid medium (containing $1.5 \% w / v$ agar) and MacConkey agar medium (BioMèrieux, Marcy l'Etoile, Francia) at $37^{\circ} \mathrm{C}$ for 24 h. K. pneumoniae subsp. pneumoniae DSMZ30104 ${ }^{\mathrm{T}}$, K. pneumoniae subsp. rhinoscleromatis DSMZ16231 ${ }^{\mathrm{T}}$, K. pneumoniae subsp. ozaenae DSMZ16358 ${ }^{\mathrm{T}}$, K. pneumoniae CECT 142, K. pneumoniae CECT 517 and K. oxytoca CECT $860^{\mathrm{T}}$ were used as reference strains.

Table 1. Biochemical and molecular identification (based on partial rpoB gene sequencing) of commensal, communityacquired, NICU outbreak and reference strains used in this study.

\begin{tabular}{|c|c|c|c|c|}
\hline Strains & Biochemical ID & $r p o B$ (\% Identity) & Origin & Source * \\
\hline \multicolumn{5}{|l|}{ Commensal } \\
\hline HA001 & K. oxytoca & K. michiganensis (100) & Faeces & UCM \\
\hline HA009 & K. oxytoca & K. michiganensis (100) & Faeces & UCM \\
\hline HI2-45 & K. oxytoca & K. michiganensis (100) & Faeces & UCM \\
\hline HV1-02 & K. oxytoca & K. michiganensis (99.52) & Faeces & UCM \\
\hline HV1-11 & K. oxytoca & K. michiganensis (99.52) & Faeces & UCM \\
\hline HV2-03 & K. oxytoca & K. michiganensis (99.52) & Faeces & UCM \\
\hline HV2-11 & K. oxytoca & K. michiganensis (99.52) & Faeces & UCM \\
\hline LG5-52 & K. oxytoca & K. michiganensis (99.52) & Milk & UCM \\
\hline MV11 & K. oxytoca & K. michiganensis (99.52) & Meconium & UCM \\
\hline LMV2-9 & K. pneumoniae & K. pneumoniae (99.76) & Milk & UCM \\
\hline LMV6-5 & K. pneumoniae & K. pneumoniae (99.76) & Milk & UCM \\
\hline LMV90-10 & K. pneumoniae & K. pneumoniae $(100)$ & Milk & UCM \\
\hline LMV90-11 & K. pneumoniae & K. pneumoniae (100) & Milk & UCM \\
\hline MV3-1 & K. pneumoniae & K. variicola (100) & Faeces & UCM \\
\hline MV31-21 & K. pneumoniae & K. pneumoniae (99.76) & Faeces & UCM \\
\hline MV91-1 & K. pneumoniae & K. pneumoniae (99.76) & Faeces & UCM \\
\hline MV91-24 & K. pneumoniae & $\begin{array}{c}\text { K. quasipneumoniae } \\
\text { subsp. similipneumoniae (100) }\end{array}$ & Faeces & UCM \\
\hline MV91-25 & K. pneumoniae & K. pneumoniae (99.76) & Faeces & UCM \\
\hline MV91-28 & K. pneumoniae & K. pneumoniae (100) & Faeces & UCM \\
\hline MV91-42 & K. pneumoniae & $\begin{array}{c}\text { K. quasipneumoniae } \\
\text { subsp. quasipneumoniae (99.52) }\end{array}$ & Faeces & UCM \\
\hline \multicolumn{5}{|c|}{ Community-acquired } \\
\hline Ko1 & K. pneumoniae & R. planticola $(100)$ & Blood culture & RYC \\
\hline Ko2 & K. oxytoca & K. michiganensis (99.76) & Blood culture & RYC \\
\hline Ko3 & K. oxytoca & K. michiganensis (99.52) & Blood culture & RYC \\
\hline Ko4 & K. oxytoca & K. michiganensis (100) & Blood culture & RYC \\
\hline Ko5 & K. oxytoca & K. michiganensis (100) & Blood culture & RYC \\
\hline Ko6 & K. oxytoca & K. michiganensis (99.76) & Blood culture & RYC \\
\hline Ko7 & K. oxytoca & K. oxytoca $(100)$ & Blood culture & RYC \\
\hline Ko8 & K. oxytoca & K. grimontii (98.80) & Blood culture & RYC \\
\hline Ko9 & K. oxytoca & K. pneumoniae (99.76) & Blood culture & RYC \\
\hline Ko10 & K. oxytoca & R. ornithinolytica (100) & Blood culture & RYC \\
\hline Ko11 & K. oxytoca & K. grimontii (99.04) & Blood culture & RYC \\
\hline Ko12 & K. oxytoca & K. michiganensis (99.52) & Blood culture & RYC \\
\hline Kp1 & K. pneumoniae & K. pneumoniae (99.76) & Blood culture & RYC \\
\hline
\end{tabular}


Table 1. Cont.

\begin{tabular}{|c|c|c|c|c|}
\hline Strains & Biochemical ID & rpoB (\% Identity) & Origin & Source * \\
\hline Kp2 & K. pneumoniae & K. pneumoniae (99.52) & Blood culture & RYC \\
\hline Kp3 & K. pneumoniae & K. pneumoniae (99.76) & Blood culture & RYC \\
\hline Kp4 & K. pneumoniae & K. pneumoniae (99.76) & Blood culture & RYC \\
\hline Kp5 & K. pneumoniae & K. pneumoniae (99.76) & Blood culture & RYC \\
\hline Kp6 & K. pneumoniae & K. pneumoniae (100) & Blood culture & RYC \\
\hline Kp7 & K. pneumoniae & K. pneumoniae (99.76) & Blood culture & RYC \\
\hline Kp8 & K. pneumoniae & K. pneumoniae (100) & Blood culture & RYC \\
\hline Kp9 & K. pneumoniae & K. pneumoniae (99.76) & Blood culture & RYC \\
\hline Kp10 & K. pneumoniae & K. pneumoniae (99.76) & Blood culture & RYC \\
\hline Kp12 & K. pneumoniae & K. pneumoniae (99.76) & Blood culture & RYC \\
\hline Kp13 & K. pneumoniae & K. pneumoniae (99.76) & Blood culture & RYC \\
\hline Kp14 & K. pneumoniae & K. pneumoniae (99.76) & Blood culture & RYC \\
\hline Kp15 & K. pneumoniae & K. pneumoniae (100) & Blood culture & RYC \\
\hline \multicolumn{5}{|l|}{ NICU outbreak } \\
\hline $\mathrm{K} 12-1$ & K. pneumoniae & K. pneumoniae (100) & Blood culture & HUDO \\
\hline $\mathrm{K} 12-2$ & K. pneumoniae & K. pneumoniae (100) & Blood culture & HUDO \\
\hline $\mathrm{K} 12-3$ & K. pneumoniae & K. variicola (99.52) & NICU environment & HUDO \\
\hline K12-4 & K. pneumoniae & K. pneumoniae (99.76) & NICU environment & HUDO \\
\hline K12-5 & K. pneumoniae & K. michiganensis (100) & Faeces & HUDO \\
\hline $\mathrm{K} 12-6$ & K. pneumoniae & K. pneumoniae (100) & Vascular catheter & HUDO \\
\hline K12-7 & K. oxytoca & K. michiganensis (100) & Faeces & HUDO \\
\hline K12-8 & K. pneumoniae & K. pneumoniae (99.76) & Faeces & HUDO \\
\hline K12-9 & K. pneumoniae & K. pneumoniae (99.76) & Faeces & HUDO \\
\hline $\mathrm{K} 12-10$ & K. pneumoniae & K. pneumoniae (99.76) & Faeces & HUDO \\
\hline \multicolumn{5}{|c|}{$\begin{array}{l}\text { Reference type strains } \\
\qquad\left({ }^{\mathrm{T}}\right)\end{array}$} \\
\hline DSM $30104^{\mathrm{T}}$ & $\begin{array}{c}\text { K. pneumoniae } \\
\text { subsp. pneumoniae }\end{array}$ & K. pneumoniae (100) & Unknown & DSMZ \\
\hline CECT 142 & $\begin{array}{c}\text { K. pneumoniae } \\
\text { subsp. pneumoniae }\end{array}$ & K. pneumoniae (99.76) & Unknown & СЕCT \\
\hline СЕСТ 517 & $\begin{array}{c}\text { K. pneumoniae } \\
\text { subsp. pneumoniae }\end{array}$ & K. pneumoniae (100) & Urine & CECT \\
\hline DSM $16231^{\mathrm{T}}$ & $\begin{array}{l}\text { K. pneumoniae } \\
\text { subsp. } \\
\text { rhinoscleromatis }\end{array}$ & K. pneumoniae (100) & Nose rhinoscleroma & DSMZ \\
\hline DSM $16358^{\mathrm{T}}$ & $\begin{array}{l}\text { K. pneumoniae } \\
\text { subsp. ozaenae }\end{array}$ & K. pneumoniae (100) & Nose & DSMZ \\
\hline CECT $860^{\mathrm{T}}$ & K. oxytoca & K. oxytoca $(100)$ & Pharyngeal tonsil & CECT \\
\hline
\end{tabular}

Abbreviations: * UCM, group 920080, Complutense University of Madrid; RYC, Hospital Universitario Ramón y Cajal; HUDO, Hospital Universitario 12 de Octubre; CECT, Spanish Type Culture Collection; DSMZ, German Collection of Microorganisms and Cell Cultures $\mathrm{GmbH}$.

\subsection{Molecular Identification of Isolates}

All isolates were re-identified by sequencing a 454-bp fragment of the rpoB gene amplified by PCR with KrpoB-for and KrpoB-rev primer pair (Table S1). PCR conditions were as follows: 1 cycle of $94{ }^{\circ} \mathrm{C}$ for $4 \mathrm{~min}, 30$ cycles of $94{ }^{\circ} \mathrm{C}$ for $30 \mathrm{~s}, 55^{\circ} \mathrm{C}$ for $30 \mathrm{~s}$ and $72{ }^{\circ} \mathrm{C}$ for $30 \mathrm{~s}$ and a final extension of $72{ }^{\circ} \mathrm{C}$ for $5 \mathrm{~min}$. Amplicons were purified using the Nucleospin Extract II kit (Macherey-Nagel, Düren, Germany) and sequenced (ABI Prism 3730; Applied Biosystems, Foster City, CA, USA) at the Genomics Unit of the Universidad Complutense de Madrid (Madrid, Spain). The resulting sequences were used to search against reference sequences deposited in the EMBL database using BLASTn algorithm (http: / / www.ncbi.nlm.nih.gov; accessed on 7 July 2021). The identity of the isolates was determined on the basis of the highest scores. The $r p o B$ gene sequences obtained were deposited in the GenBank database, under the accession numbers KJ499842 to KJ499903. 


\subsection{Phylogenetic Analysis}

For phylogenetic analysis, the $r p o B$ sequences obtained were aligned by using the Clustal W method [18] with the MEGA version 6 (Revision 6.06, update February 2014) software created by Tamura, Stecher, Peterson, Filipski, and Kumar [19]. Phylogenetic trees were constructed based on the neighbour-joining method with the Jukes-Cantor parameter model [20]. Bootstraping analysis (1000 replicates) was performed to study the stability of the groupings. The $r p o B$ sequences of the following type species were obtained from the GenBank database and included in the phylogenetic analysis the following: K. variicola

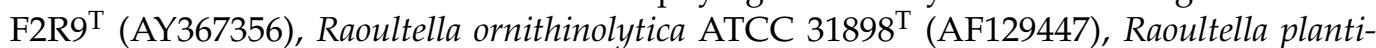
cola ATCC $33531^{\mathrm{T}}$ (AF129449) and Staphylococcus sciuri subsp. carnaticus ATCC $700058^{\mathrm{T}}$ (DQ120748).

\subsection{Genotyping of Isolates}

Pulsed-field gel electrophoresis (PFGE) was performed as described previously [21]. Chromosomal DNA of each isolate was digested with $30 \mathrm{U}$ of XbaI (TaKaRa Bio Inc, Shiga, Japan). Electrophoresis was carried out in a CHEF DR II apparatus (Bio-Rad, Laboratories, Hercules, CA, USA) with the following conditions: $14{ }^{\circ} \mathrm{C}, 6 \mathrm{~V} / \mathrm{cm}^{2}$ and $10-40 \mathrm{~s}$ for 24 $\mathrm{h}$. Dendrograms of genetic relationships were constructed by using the Phoretix 1D software (version 5.0; Nonlinear Dynamics Ltd., Newcastle upon Tyne, UK) based on the Dice coefficient.

Small plasmids were extracted from $16 \mathrm{~h}$ BHI broth cultures with the "QIAprep Spin Miniprep Kit" (QIAgen) as recommended by the manufacturer and visualized in a $1 \%$ agarose gel by using conventional electrophoresis ( $90 \mathrm{~V}$ for $90 \mathrm{~min}$ ). Supercoiled ladder (1-16 kb) (Invitrogen, Paisley, UK) was used as molecular weight marker. Large plasmids $(>16 \mathrm{~kb})$ were extracted using PFGE-S1 nuclease digestion (Takara Bio Inc, Shiga, Japan) with the following conditions: $14{ }^{\circ} \mathrm{C}, 6 \mathrm{~V} / \mathrm{cm}^{2}$ and $5-25 \mathrm{~s}$ for $3 \mathrm{~h}$ followed by $30-45 \mathrm{~s}$ for $12 \mathrm{~h}$. Size was determined using the Lambda Ladder PFG Marker (48.5-1000, $18 \mathrm{~Kb}$ ) and Low Range PFG Marker (0.13-194 Kb) (New England Biolabs, Inc.) as references. The resulting plasmid profiles were graphically represented and analysed using the software available on http:/ /insilico.ehu.es/dice_upgma/ (accessed on 7 July 2021) to generate dendrograms by UPGMA clustering using Dice correlation.

\subsection{Antimicrobial Susceptibility Testing}

Minimal inhibitory concentrations (MICs) to antibiotics were evaluated by a microdilution method using the Sensititre EMIZA 9EF (TREK Diagnostic Systems, Cleveland, EEUU) plates following the manufacturer's instructions. Production of Extendedspectrum $\beta$-lactamases (ESBLs) was tested by the double-disk synergy test [22] containing ceftazidime/ceftazidime plus clavulanate or cefotaxime/cefotaxime plus clavulanate. The presence of CTX-M $\beta$-lactamase-encoding genes $\left(b l a_{\mathrm{CTX}} \mathrm{M}\right)$ was identified by multiplex PCR using the oligonucleotides CTX-M-1G-F, CTX-M-1G-R, CTX-M-2G-F, CTX-M-2G-R, CTX-M-9G-F and CTX-M-9G-R (Table S1) and conditions described previously [23]. P1 and P2b primers (Table S1) were used to amplify bla $a_{\mathrm{CTX}-\mathrm{M}}$ subgroup I genes [24]. Multidrugresistance (MDR) was defined as non-susceptibility (resistance) to at least one agent in three or more antimicrobial categories [25].

\subsection{Virulence Determinants}

Presence of the magA, rmpA, wabG, uge, kfu and fimH genes encoding potential virulence factors was determined by PCR using specific primers (Table S1) and conditions described previously [26,27]. A novel multiplex PCR was designed to detect genes associated to biosynthesis or receptors of the siderophores aerobactin (iut $A, i u c B)$, enterobactin $(f e p A, f e p C)$ and yersibactin $(f y u A, y b t T)$. For this purpose, six oligonucleotide pairs were designed (iutA-F/iutA-R, iucB-F/iucB-R, fepA-F/fepA-R, fepC-F/fepC-R, FyuA-F/FyuA$\mathrm{R}$ and YbtT-F/YbtT-R; Table S1), which result in the amplification of DNA fragments of 580, 692, 897, 280, 828 and 451 bp, respectively. PCR conditions were as follows: 1 cycle 
of $94{ }^{\circ} \mathrm{C}$ for $4 \mathrm{~min}, 30$ cycles of $94{ }^{\circ} \mathrm{C}$ for $30 \mathrm{~s}, 62{ }^{\circ} \mathrm{C}$ for $30 \mathrm{~s}, 72{ }^{\circ} \mathrm{C}$ for $1 \mathrm{~min}$ and a final extension of $72{ }^{\circ} \mathrm{C}$ for $5 \mathrm{~min}$.

\subsection{Hypermucoviscosity, Biofilms, Siderophores and Bacteriocin Activity Assays}

The hypermucoviscous phenotype was determined by the string test [28]. The biofilm formation ability was analysed in polyvinylchloride plastic (PVC) microtiter plates as described previously [29,30]. Siderophores production was quantified in cell-free supernatants [31]. The ability to inhibit the growth of other strains by production of bacteriocinlike substances was tested both on solid and broth medium, according to the direct [32] and the "spot-on-the-lawn" methods [33], respectively.

\section{Results}

\subsection{Molecular Identification of Klebsiella Species}

The amplification and sequencing of a $454 \mathrm{bp}$ fragment of the $r p o B$ gene achieved a great level of discrimination of the Klebsiella isolates at the species level when compared against the $r p o B$ sequences from the validated type strains (Table 1). Among the 21 isolates initially identified as K. oxytoca by routine biochemical methods, just 1 isolate was identified as $K$. oxytoca sensu stricto by rpoB sequencing. The rest of the isolates were identified as K. michiganensis $(n=16), K$. grimontii $(n=2)$, K. pneumoniae $(n=1)$ and $R$. ornithinolytica $(n=1)$. In the case of the 35 Klebsiella isolates initially identified as K. pneumoniae by biochemical methods, most of them were identified as K. pneumoniae sensu stricto $(n=29)$ based on $r p o B$ sequencing; the remaining isolates were identified as K. variicola $(n=2), K$. quasipneumoniae subsp. similipneumoniae $(n=1)$, K. quasipneumoniae subsp. quasipneumoniae $(n=1), K$. michiganensis $(n=1)$ and Raoultella planticola $(n=1)$. The reference strains K. pneumoniae subsp. pneumoniae DSMZ30104 ${ }^{\mathrm{T}}$, K. pneumoniae subsp. rhinoscleromatis DSMZ16231T, K. pneumoniae subsp. ozaenae DSMZ16358 ${ }^{\mathrm{T}}$, K. pneumoniae CECT 142, K. pneumoniae CECT 517 and K. oxytoca CECT $860^{\mathrm{T}}$ were correctly identified to the species level by rpoB sequencing.

\subsection{Phylogenetic Analysis Based on rpoB}

The phylogenetic analysis based on the partial amplification of the rpoB gene, supported the molecular identification of all Klebsiella isolates. Indeed, all K. pneumoniae isolates clustered with phylogenetic group KpI (K. pneumoniae), while isolates MV91-24 and MV91-42 clustered with KpII-A (K. quasipneumoniae subsp. similipneumoniae) and KpII-B (K. quasipneumoniae subsp. quasipneumoniae), respectively. The MV3-1 and K12-3 isolates clustered with KpIII (K. variicola subsp. varicola). All isolates identified as K. michiganensis clustered with phylogroup KoI (K. michiganensis), while isolate Ko7 clustered with KoII (K. oxytoca) and isolates Ko8 and Ko11 clustered with KoIV (K. grimontii). The two identified Raoultella isolates, Ko1 and Ko10, clustered with R. planticola and with R. ornithinolytica, respectively (Figure 1).

\subsection{Genetic Diversity}

A high genetic diversity among the K. pneumoniae and the K. oxytoca complex isolates was detected by PFGE (Figure S1). However, within the K. pneumoniae isolates, two clonal groups (>80\% similarity) were identified: CP1, formed by K. pneumoniae K12-1, K12-6 and K12-8, and CP2, formed by K. pneumoniae K12-4 and K12-9 (Figure S1A). All these clonal isolates were isolated from the NICU's outbreak. In the K. oxytoca complex, we identified two clonal groups (CM1 and CM2), formed by K. michiganensis HA001 and HA009, and K. michiganensis HV1-02 and HV2-11, respectively (Figure S1B). Those isolates were isolated from faeces from breast-fed healthy term infants.

Plasmid profiles showed that a total of $28(89 \%)$ isolates from the K. pneumoniae complex (Figure S2A) and 18 (85\%) from the K. oxytoca complex (Figure S2B) contained plasmids (1 to 8 plasmids, ranging from 1 to $600 \mathrm{~kb}$ ). 


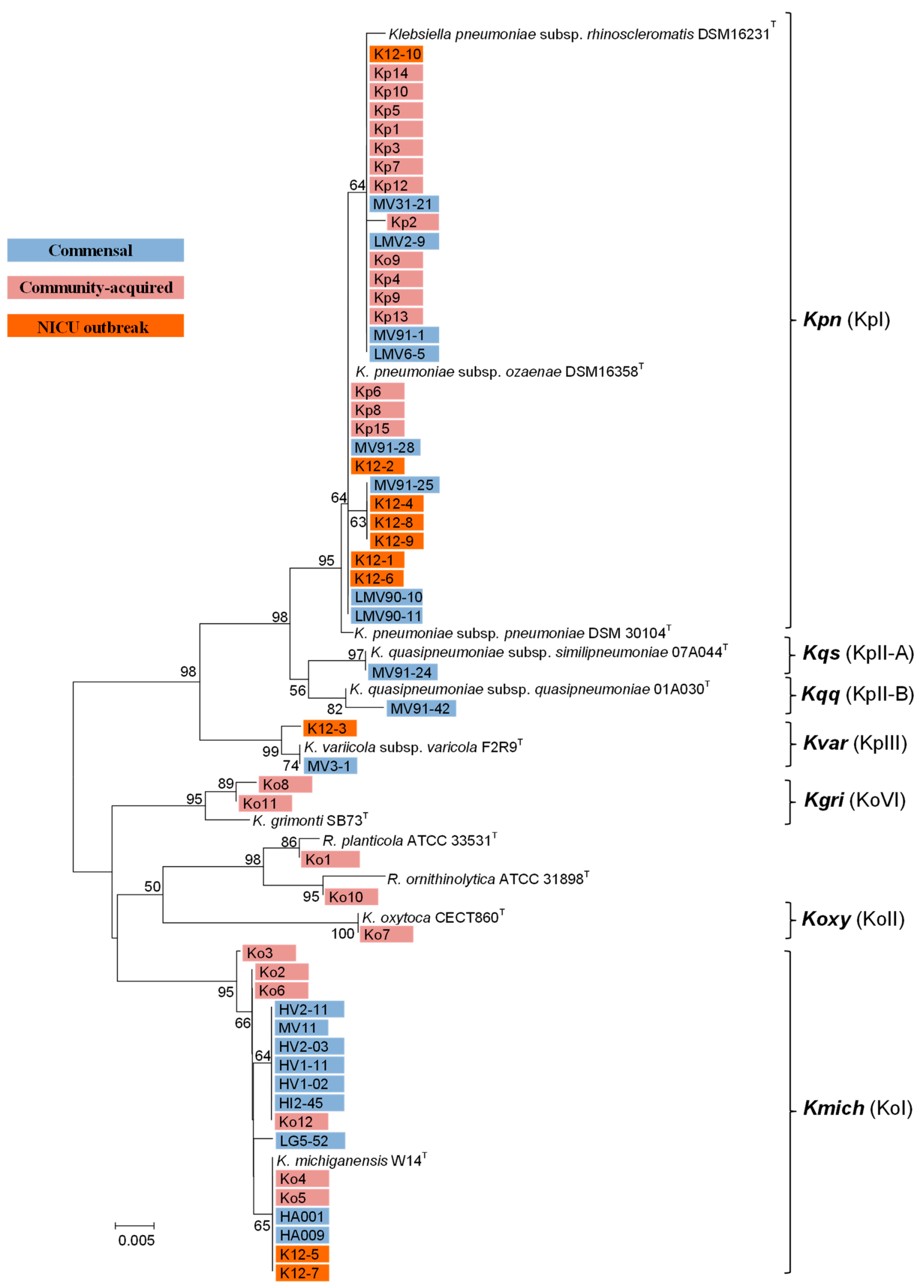

Figure 1. Phylogenetic relationships of 56 Klebsiella isolates and reference type strains by comparison of partial rpoB gene sequences (454 bp): The tree was based on the neighbour-joining method, using the Jukes-Cantor parameter model. Numbers on the tree indicate bootstrap values calculated for 1000 subsets for branch-points greater than 50\%. Bar, 0.005 nucleotide changes per nucleotide position. Phylogenetic groups (KpI, KpII-A, KpII-B, KpIII, KoII, KoVI and KoI) are shown in brackets (Kpn: K. pneumoniae; Kqs: K. quasipneumoniae subsp. similipneumoniae; Kqq: K. quasipneumoniae subsp. quasipneumoniae; Kvar: K. variicola; Kgri: Klebsiella grimontii; Koxy: K. oxytoca; Kmich: K. michiganensis). 


\subsection{Antimicrobial Susceptibility}

Low antibiotic resistance rates were detected in both commensal and bacteraemic community-acquired collections (Figure 2). The isolates were susceptible to most antibiotics tested with the exception of K. quasipneumoniae subsp. similipneumoniae MV91-24, an MDR and imipenem-resistant isolate from faeces of a healthy breast-fed infant, and K. pneumoniae Kp9 and K. michiganensis Ko12, two MDR isolates from community-acquired infections.

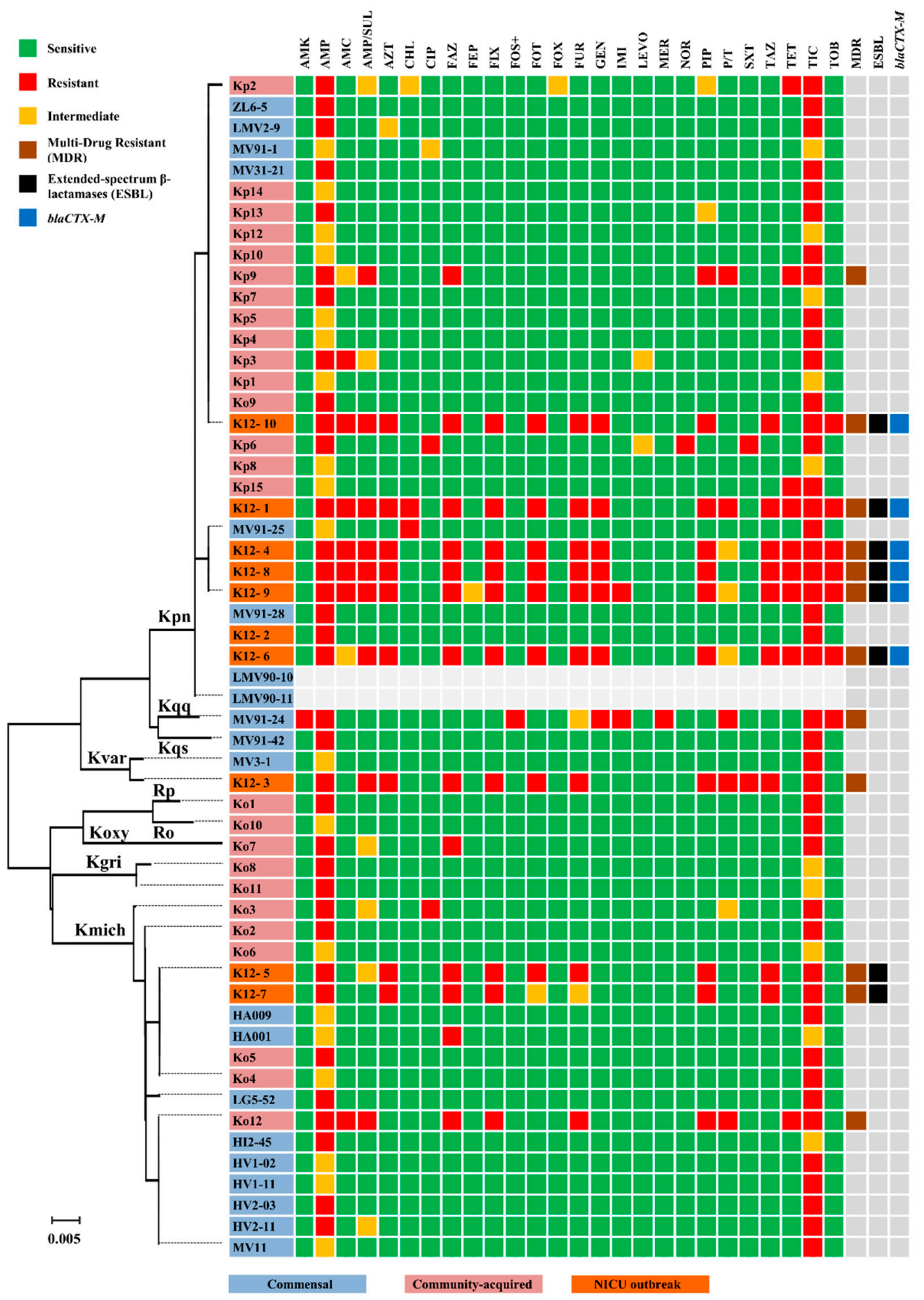

Figure 2. Antibiotic resistance profiles among the Klebsiella isolates analysed in this study: Antibiotics: amikacin (AMK), ampicillin (AMP), amoxicillin-clavulanic acid (AMC), ampicillin-sulbactam (AMP/SUL), aztreonam (AZT), chloramphenicol (CHL), ciprofloxacin (CIP), cefazolin (FAZ), cefepime (FEP), cefixime (FIX), fosfomycin (FOS), cefotaxime (FOT), cefoxitin (FOX), cefuroxime (FUR), gentamicin (GEN), imipenem (IMI), levofloxacin (LEVO), meropenem (MER), norfloxacin (NOR), piperacillin (PIP), piperacillin-tazobactam (P/T), trimethoprim-sulfamethoxazole (SXT), ceftazidime (TAZ), tetracycline (TET), ticarcillin (TIC) and tobramycin (TOB). 
All isolates from the NICU showed a MDR phenotype, including ESBL production, with the unique exception of K. pneumoniae K12-2 (Figure 2). The antibiotic susceptibility profiles of K. pneumoniae K12-1, K12-4, K12-6, K12-8, K12-9 and K12-10, K. michiganensis K12-5 and K12-7 and K. variicola K12-3 were compatible with ESBLs production, a fact that was subsequently confirmed by the double-disk synergy test and the presence of the blactX-M-15 gene.

\subsection{Virulence Determinants, Hypermucoviscosity, Biofilms and Siderophores}

The presence of the wabG, uge and kfu genes was detected in $38.2 \%, 38.2 \%$ and $20.6 \%$ of the isolates from the K. pneumoniae complex, respectively (Figure 3). The wabG gene was more represented among commensal than among community-acquired isolates $(p<0.05)$, while the uge gene was more abundant among the NICU outbreak isolates than among the community-acquired ones $(p>0.05)$. All members of the K. pneumoniae complex presented the fimH gene (with the exception of K. pneumoniae Ko9), whereas the magA and rmpA genes were not detected. In contrast, none of the isolates from the K. oxytoca complex contained any of the virulence genes studied by PCR.

A novel multiplex PCR targeting the biosynthesis and receptor genes of the siderophores aerobactin, enterobactin and yersibactin (Figure 3) showed that among the K. pneumoniae complex, $64.7 \%$ presented the fepA gene (enterobactin synthesis), $79.4 \%$ the fepC (enterobactin receptor), $14.7 \%$ the fyuA (yersibactin synthesis) and $14.7 \%$ the ybtT (yersibactin receptor). None of the isolates harboured the genes iutA and iucB encoding the synthesis and receptor genes of aerobactin. The fepC gene was more represented among commensal than among community-acquired isolates $(p<0.05)$.

Within the K. oxytoca complex, the fepA gene was not detected in any of the isolates, while the fepC gene was present in $55 \%$ of the isolates. The genes fyuA and ybtT were detected in $55 \%$ and $5 \%$ of the isolates. The fyuA gene was less represented among community-acquired than among commensal isolates $(p<0.05)$. The iucB gene encoding the aerobactin receptor was only detected in two isolates.

None of the Klebsiella isolates showed a hypermucoviscous phenotype. Only $14.7 \%$ of the isolates from the K. pneumoniae complex (all belonging to K. pneumoniae) were able to grow on biofilms formation on PVC plates (Figure 3), while none of the isolates from the K. oxytoca group showed this property. No significant differences were found in the ability to produce siderophores when comparing the isolates from the K. pneumoniae and K. oxytoca complex. However, commensal isolates produced more siderophores than community-acquired isolates $(p>0.05)$.

\subsection{Antimicrobial Activity}

Antimicrobial activity was only produced by the commensal K. pneumoniae MV91-1 and the community-acquired K. pneumoniae Kp5 isolates (Figure 4). MV91-1 showed a wide inhibitory spectrum against the other Klebsiella isolates, being active against K. pneumoniae, K. quasipneumoniae subsp. similipneumoniae, K. pneumoniae subsp. quasipneumoniae, K. variicola, R. ornithinolytica, K. grimontii and K. michiganensis (Figure 3). In contrast, the inhibitory spectrum of Kp5 was narrower, being active only against $K$. pneumoniae, K. variicola and K. michiganensis (Figure 3). The inhibitory activity of both strains was displayed only on solid medium and was abolished after the addition of proteinase $\mathrm{K}(1 \mathrm{mg} / \mathrm{mL}$ final concentration). 
Siderophores (High)

Siderophores (Medium)

Siderophores (Low)

Biofilms formation

Bacteriocins production

Sensitivity to K. pneumoniae MV91-1

Sensitivity to $K$. pneumoniae KP5

Z Not tested

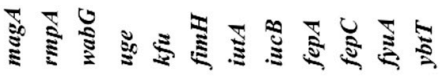

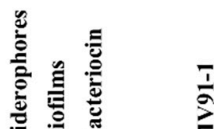

$\frac{1}{2}$

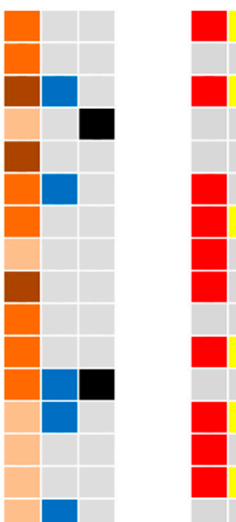

\section{ZL6-5}

LMV2-9

MV91-1

MV31-21

Kp14

Kp13

Kp12

Kp10

Kp9

Kp7

Kp5

Kp4

Kp3

Kp1

Ko9

K12-10

Kp6

Kp8

Kp15

K12-1

MV91-25

K12- 4

K12-8

$\begin{array}{r}\text { K12- } 8 \\ \text { K12- } 9 \\ \hline\end{array}$

MV91-28
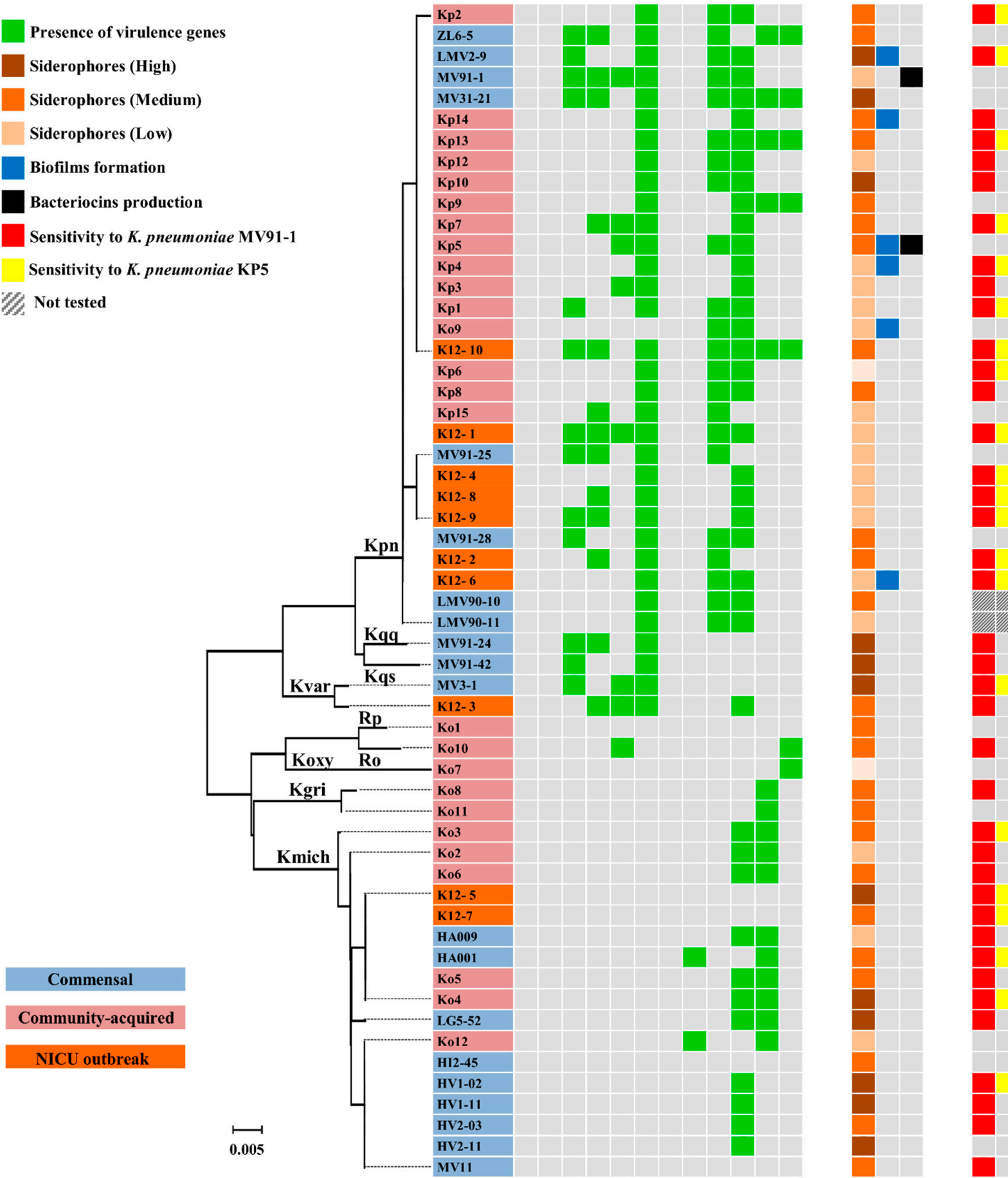

Figure 3. Virulence genes and phenotypic features of the Klebsiella isolates analysed in this study: The presence of genes involved in virulence, i.e., $\operatorname{mag} A$ and $\operatorname{rmp} A$ (hypermucoviscosity phenotype), wabG (lipopolysaccharide synthesis), uge (uridine diphosphate galacturonate 4-epimerase), $k f u$ (iron-uptake system), fimH (type 1 fimbrial adhesin), iut $A$ (siderophore Aerobactin), iut B (receptor of Aerobactin), fepA (siderophore Enterobactin), fepC (receptor of Enterobactin), fyuA (siderophore Yersibactin), ybtT (receptor of Yersibactin), was determined by PCR. Production of siderophores was quantified in cellfree supernatants; biofilm formation was assayed and quantified in PVC microtiter plates; production of proteinaceous antimicrobial compounds (bacteriocins) was tested on solid culture medium against all Klebsiella isolates of this study, used as indicator strains. Sensitivity to bacteriocins produced by K. pneumoniae MV91-1 and Kp5 was assayed on solid medium. 
MV91-1
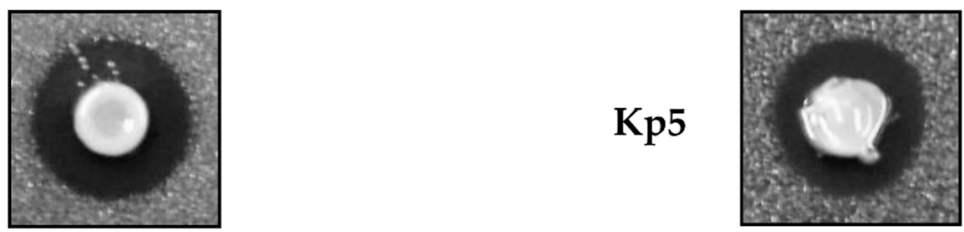

Figure 4. Antimicrobial activity of K. pneumoniae MV91-1 (commensal) and Kp5 (communityacquired) against K. pneumoniae K12-9, a multidrug ESBL-producer isolate from NICU.

\section{Discussion}

Species from the genus Klebsiella, such as K. pneumoniae or K. oxytoca, are well-known for their ability to cause a wide range of infections in humans, some of them with fatal consequences [1]. An additional concern is that Klebsiella spp. has readily developed antimicrobial resistance to multiple antibiotics, being difficult to treat and eliminate with current antibiotics, thus generating a serious threat to public health [34]. Although most studies have been focused on the study of Klebsiella isolates from clinical samples (nosocomial and community-acquired infections), recent studies have shown that our own gastrointestinal tract is a potential reservoir of Klebsiella isolates, making difficult the delimitation between pathogens and commensal isolates [10-13]. This, together with the increasing number of novel Klebsiella species associated with humans that could cause severe infections, requires the development of new studies focused on the identification and characterization of commensal Klebsiella isolates from healthy subjects.

A proper identification of Klebsiella isolates at the species level is relevant from an epidemiological and clinical point of view, but routine biochemical tests have limitations since members of this genus possess similar biochemical and phenotypic features. Molecular identification based on ribosomal $16 \mathrm{~S}$ rDNA gene sequences has been useful in defining bacterial relationships, including those of Klebsiella [35]; however, their value for delineating closely related species seems limited because of the scarce nucleotide variation. The identification of Klebsiella species and phylogenetic groups within K. pneumoniae and K. oxytoca based on molecular methods can now be reliably achieved based on the sequencing of housekeeping genes such as rpoB, gyr $A$ and parC $[4,5,36]$.

In this work, we have shown that the amplification and sequencing of a $454 \mathrm{bp}$ fragment of the rpoB gene achieved a great level of discrimination for the identification of Klebsiella isolates, which was supported by the phylogenetic analysis, thus providing a fast and reliable tool to identify species belonging to this genus. Among the 35 isolates identified as K. pneumoniae by biochemical methods, 29 were identified as K. pneumoniae sensu stricto based on $r p o B$, while the remaining 6 corresponded to K. variicola $(n=2)$, K. quasipneumoniae subsp. similipneumoniae $(n=1), K$. quasipneumoniae subsp. quasipneumoniae $(n=1)$, K. michiganensis $(n=1)$ and R. planticola $(n=1)$ (Table 1). Although most K. pneumoniae were correctly identified by routine biochemical approaches, the misidentification of $K$. variicola and K. quasipneumoniae as K. pneumoniae is according to the results provided by previous studies $[5,7,8,37]$. This should be carefully considered since K. variicola and K. quasipneumoniae can cause severe life-threatening infections similar to K. pneumoniae $[7,8]$. On the other hand, most of the isolates identified by biochemical methods as K. oxytoca (16 of 21) were identified as $K$. michiganensis based on $r p o B$, while the remaining 5 were classified as K. grimontii $(n=2)$, K. pneumoniae $(n=1), K$. oxytoca sensu stricto $(n=1)$ and R. ornithinolytica $(n=1)$. Thus, just 1 of the 21 isolates identified as K. oxytoca by routine biochemical approaches was K. oxytoca sensu stricto, while the vast majority belonged to K. michiganensis. These results support the findings of a recent study that have suggested that $K$. michiganensis could be more clinically relevant than $K$. oxytoca in human-associated infections [8]. This misidentification could have hidden the actual clinical and epidemiological significance of these species and, therefore, the implementation of alternative taxonomic methods, such as the sequencing of the $r p o B$ gene, should be encouraged in clinical microbiology laboratories. 
Interestingly, we found a great variety of commensal Klebsiella spp. isolated from healthy subjects (milk from lactating women and faeces from breast-fed infants), such as K. pneumoniae, K. quapsineumoniae subsp similipneumoniae and K. quapsineumoniae subsp quasipneumoniae, K. variicola and K. michiganensis. This is in line with a recent study where it has been described that "healthy" antibiotic-treated preterm infants hospitalized in NICUs can harbour different Klebsiella species, including K. pneumoniae, K. quasipneumoniae, K. grimontii and K. michiganensis [8]. Similarly, K. pneumoniae and K. oxytoca have been described as components of the human gut microbiota from healthy adults [9-11]. With respect to community-acquired infections, we also found a great variety of species, such as K. pneumoniae, K. oxytoca, K. michiganensis, K. grimontii, R. planticola and R. ornithinolytica. Although Raoultella spp. are infrequent human pathogens, $R$. ornithinolytica is considered as emerging bacteria causing human infections [38,39]. The low prevalence of $R$. ornithinolyticaand $R$. planticola-related infections could be due to their misidentification as K. pneumoniae or K. oxytoca by conventional biochemical tests. In relation to the isolates from the NICU outbreak, we found two K. michiganensis strains (K12-5 and K12-7) that had been misidentified as K. pneumoniae and K. oxytoca, respectively. A recent study reported, for the first time, an NICU nosocomial outbreak caused by MDR and ESBL-producing $K$. michiganensis isolates [40]. This could indicate that hospital outbreaks caused by K. michiganensis could have passed undetected due their misclassification as other Klebsiella species. In addition, we found that an NICU's isolate (K12-3) previously misidentified as K. pneumoniae actually belonged to $K$. variicola, which is currently considered as an emerging human pathogen $[7,41]$

A high genetic diversity was found among the commensal and the communityacquired Klebsiella isolates, whereas those from the NICU seemed to be the result of a polyclonal dissemination. Globally, the commensal and community-acquired Klebsiella isolates were sensitive to most of the antibiotics assayed in this study, with the exception of the commensal MV91-24 (K. quasipneumoniae subsp. similipneumoniae), and the communityacquired Ko12 (K. michiganensis) and Kp9 (K. pneumoniae), which were multi-drug-resistant (MDR). In contrast, 9 out of the 10 Klebsiella isolates from the NICU were MDR, thus supporting the situation of nosocomial outbreak. In addition, the NICU bla $a_{\mathrm{CTX}-\mathrm{M}-15 \text {-producing }}$ K. pneumoniae K12-9 and the commensal K. quasipneumoniae MV91-24 isolates exhibited resistance to imipenem, thus indicating the wide dispersion of carbapenemases, either combined with ESBL or not, among different environments, a fact that has been described previously $[42,43]$.

ESBL-producing K. pneumoniae can cause infection outbreaks in NICUs. In this study, some of the strains were isolated from blood cultures (K. pneumoniae K12-1 and K12-2), vascular catheters (K. pneumoniae K12-6), faeces (K. michiganensis K12-5 and K127 and K. pneumoniae K12-8, K12-9 and K12-10) or the environment (K. variicola K12-3 and $K$. pneumoniae K12-4) during the investigation of a neonatal-ICU outbreak. Most of them were MDR and ESBL-producing strains, despite the fact that they belonged to different Klebsiella species, thus suggesting the existence of a polyclonal outbreak in the NICU. The six K. pneumoniae isolates bla $a_{\mathrm{CTX}-\mathrm{M}-15}$ producers, were grouped in three phylotypes (K12-1, K12-6 and K12-8; K12-4 and K12-9; K12-10), while the two MDR K. michiganensis isolates (K12-5 and K12-7) were not genetically related (Figure S1). Thus, although many outbreaks have been associated with the dissemination of a single clone, the polyclonal dissemination of different ESBL-producing Klebsiella strains can happen in the same unit at the same time [44]. Plasmids can play an essential role in the dissemination of antibiotic resistance among clinically relevant pathogens, as it has been described previously [44-46]. In this study, we have described that Klebsiella isolates possess a variable number (from 0 to 8) of large and small plasmids ranging from 1 to $600 \mathrm{~kb}$ in size which could reflects its ability to acquire and transfer genetic determinants, including virulence genes, pathogenicity islands and antimicrobial-resistance genes. The epidemiology of the NICU outbreak would require further investigation by using genomic approaches, as it was not the primary scope of this work. 
The inverse correlation between antibiotic resistance and virulence has been previously proposed $[47,48]$. In this study, however, we did not find any correlation between antibiotic resistance and the carriage of virulence genes or the ability to produce biofilm or siderophores, despite a heterogeneity in the virulence factors being reported by other authors [37]. In the same manner, we did not observe strong correlations between the source of isolates (commensal, community acquired or NICU outbreak) and the presence of virulence traits. Similarly, little differences were found in the phenotypic and genomic characteristics of Klebsiella isolates recovered from healthy and sick infants [8].

None of the Klebsiella isolates harboured the $\operatorname{mag} A$ and $\operatorname{rmp} A$ genes, which have been associated with the hypermucoviscosity phenotype in liver-invasive strains [26,27]. This was in agreement with the phenotypic assays performed in this work since none of the Klebsiella isolates studied showed a hypermucoviscous phenotype. In contrast, all members of the K. pneumoniae complex (with the exception of K. pneumoniae Ko9) presented the fim $H$ gene encoding the type 1 fimbrial adhesion. Thus, $100 \%$ of commensal K. pneumoniae strains that were isolated from healthy subjects harboured the fim $H$ gene, which is in contrast with the results of other authors, who found that K. pneumoniae strains isolated from the gut microbiota of healthy subjects were negative for that gene [10]. Other studies, however, have described a similar proportion of the fimH gene in K. pneumoniae isolates from community-acquired bacteraemia [27,37,49].

Within the K. pneumoniae complex, the wabG gene, encoding proteins involved in lipopolysaccharide synthesis [50] was detected in $38.2 \%$ of isolates and was more represented among commensal than among community-acquired isolates. Interestingly, the $w a b G$ gene was present in nearly all strains that had been isolated from faeces. The uge gene, encoding uridine diphosphate galacturonate 4-epimerase [51], was detected in $38.2 \%$ of isolates and was more abundant among the NICU outbreak isolates than among the community-acquired ones. In a recent study on Klebsiella spp. causing community-acquired infections, it has been described a positive rate of $28.2 \%$ and $61.5 \%$ for $w a b G$ and $u g e$ genes, respectively [37], while in other studies, the rates of positivity were close to $100 \%$ for both uge and wabG among virulent clones of K. pneumoniae [49,52]. Neither wabG nor uge were present in the isolates belonging to the K. oxytoca complex.

Iron is essential for the growth of most bacterial pathogens, so the ability to acquire iron is frequently associated with bacterial virulence. The $k f u$ (iron-uptake system) gene has been associated with higher virulence in $K$. pneumoniae [26,53,54]. In our study, a low proportion of K. pneumoniae isolates carried the $k f u$ gene, similarly as was recently described by other authors [10,37]. Interestingly, the two K. variicola strains (MV3-1 and K12-3) and $R$. ornithinolytica Ko10 presented the $k f u$ gene. In addition, bacteria can obtain iron from the host by removing it via siderophore-mediated uptake systems. In our study, we designed a novel multiplex PCR to detect genes associated to biosynthesis or as receptors of the siderophores aerobactin $(i u t A, i u c B)$, enterobactin $(f e p A, f e p C)$ and yersibactin $(f y u A, y b t T)$. Within the K. pneumoniae complex, the gene encoding enterobactin $(f e p A)$ was less abundant $(64 \%)$ than the gene $f e p C$ encoding the receptor of enterobactin (79\%). In addition, the enterobactin receptor gene $(f e p C)$ was more represented in commensal than in communityacquired isolates. In line with our results, recent studies have shown that enterobactin genes are present in a high proportion of the K. pneumoniae strains isolated from healthy and diseased preterm infants [8] and from community-acquired cases [37]. In relation to yersiniabactin, the synthesis $(f y u A)$ and receptor $(y b t T)$ genes were less represented than enterobactin-related genes, but all isolates harbouring the fyuA gene also presented the $y b t T$ gene. In contrast, none of the isolates harboured the genes iut $A$ and $i u c B$ encoding the synthesis and receptor genes of aerobactin. Those results agree with other studies were the yersiniabactin and aerobactin genes were less represented than those corresponding to enterobactin [10,37]. None of the K. quapsineumonaie isolates harboured any of the siderophores genes tested, while only one isolate of $K$. variicola harboured the gene encoding the enterobactin receptor. 
Within the K. oxytoca complex, the fepA gene encoding enterobactin was not detected in any of the isolates, while the $f e p C$ gene encoding the enterobactin receptor was present in $55 \%$ of the isolates, all belonging to $K$. michiganensis strains. The gene encoding yersinibactin $(f y u A)$ was more represented in the K. oxytoca that in the K. pneumoniae complex and was only harboured by K. michiganensis and K. grimontii. However, all isolates harbouring the gene encoding yersiniabactin lacked the gene encoding its corresponding receptor $(y b t T)$, which was only detected in K. oxytoca sensu stricto. Interestingly, the fyuA gene was less represented among community-acquired than among commensal isolates $(p<0.05)$. Aerobactin was not detected in any of the isolates of the $K$. oxytoca complex, while its receptor $(i u c B)$ was only detected in two K. michiganensis isolates. The genomic analysis of K. michiganensis and K. grimontii strains isolated from preterm infants has shown the presence of the enterobactin and yersiniabactin genes and the absence of the aerobactin gene in these species [8].

Our study has shown that most of Klebsiella isolates would be able to produce enterobactin, while a much smaller percentage would produce either aerobactin or yersiniabactin. Although these results agree with previous studies [1,8,10,37], our work revealed the high proportion of Klebsiella isolates that possess the gene encoding the receptor for enterobactin but not the gene for its biosynthesis. These isolates would be able to cheat the siderophore enterobactin produced by other bacteria, which could increase their competitiveness [55]. Finally, we did not find any correlation between the phenotypic production of siderophores and the presence of siderophore genes. This could be due to the presence of other ironuptake systems different from that reported in this study, the lack of functionality of some of the genes studied by PCR or the existence of regulatory mechanisms, which would require further investigation.

The ability of the Klebsiella to produce antimicrobial compounds both in solid and liquid medium was also addressed in this work. Two isolates of K. pneumoniae, MV91-1 (commensal) and Kp5 (community-acquired), inhibited the growth of other Klebsiella strains when assayed on solid medium (Figure 4). The inhibitory activity of both strains was sensitive to proteinase $\mathrm{K}$, thus indicating that they most likely produce bacteriocins, which are traditionally defined as antimicrobial compounds of proteinaceous nature with antimicrobial activity against related species [56]. The inhibitory spectrum of K. pneumoniae MV91-1 against the collection of Klebsiella isolates included in this work was extraordinarily wide, being active against K. pneumoniae, K. quasipneumoniae subsp. similipneumoniae and subsp. quasipneumoniae, K. variicola, R. ornithinolytica, K. grimontii and K. michiganensis. In contrast, the inhibitory spectrum of K. pneumoniae Kp5 was narrower, being active only against K. pneumoniae, K. variicola and K. michiganensis. Interestingly, both K. pneumoniae MV91-1 and Kp5 were active against all the MDR and ESBL-producing isolates from the NICU's outbreak. In addition, K. pneumoniae MV91-1 was active against the imipenem-resistant K. pneumoniae K12-9 and MV91-24 isolates. The characterization of these K. pneumoniae bacteriocins would open the door to the development of new antimicrobial strategies to combat Klebsiella strains, thus reducing the burden of antimicrobial resistance. In a recent study, it has been shown that bacteriocins from Klebsiella can be used for broad and efficient control of Klebsiella pathogens, in particular against MDR isolates [57].

The presence of different Klebsiella spp. in the milk of healthy women and in faeces of healthy breast-fed infants [15-17] suggest that these species are natural inhabitants of human microbiota, as has been described previously [8-13]. Attending to the virulence factors detected, the production of siderophores and biofilms and the plasmid content (small and large plasmids) observed, commensal strains were virtually indistinguishable from community-acquired and NICU outbreak isolates. Thus, these commensal isolates have all the potential to become pathogenic and cause infectious diseases, which is line with the recent studies showing that many infections are self-acquired from the patient's own gastrointestinal microbiota $[12,13]$. The presence of virulence traits within each commensal strain could be determined by some of the conserved genes that form the core genome within each Klebsiella species, while other virulence and antibiotic resistance genes 
would be determined by a pool of accessory genes (accessory genome) that can be shared between different Klebsiella species and even between different genera [58]. Nowadays, it is becoming more evident that Klebsiella has the ability of exchanging and assembling a wide portfolio of genes that are involved in colonization, infection and antimicrobial resistance, which in the last instance would determine if a commensal strain remains asymptomatic or turns pathogenic [58]. Commensal Klebsiella isolates, which are well adapted to live and compete within the human microbiota, are prone to acquiring antibiotic resistant genes, by horizontal gene transfer, after the colonization with antibiotic-resistant Klebsiella strains acquired in hospital environments, such as an NICU, or from other host bacteria that have developed resistance after antibiotic treatments [59]. This combination (adaptation to the host and antibiotic resistance) could lead to the apparition of new MDR Klebsiella isolates that could be difficult to treat with current antibiotic-based strategies. This could be the case of the commensal isolate $K$. quapsineumoniae subsp. similipneumoniae MV91-42 (isolated from a healthy breast-fed infant) which is MDR, ESBL-producing and resistant to imipenem and that deserves further investigation.

Our results highlight the importance of studying Klebsiella spp. from the microbiota of healthy people, which could help to create surveillance programs aimed to know the prevalence of antibiotic resistance in the population. In this line, it has been proposed that the screening of the patient's gut microbiota after their admission to hospital could help to guide the application of the correct treatment [60]. Finally, we should increase our efforts in the development of novel alternatives or complementary strategies to antibiotics, which could help to reduce AMR in bacteria. These strategies could include the use of live biotherapeutic products, which has been demonstrated to be a real alternative to the use of antibiotics to combat infectious diseases [61] or the use of bacteriocins, such as plantaricin NC8 [62], as adjuvant in combination therapy to potentiate the effects of antibiotics and reduce their overall use [63]. The fact that the commensal isolate K. pneumoniae MV91-1 was able to inhibit the growth of the MDR, ESBL-producing, imipenem-resistant strains K12-9 and MV91-24, deserves to be further explored.

\section{Conclusions}

By sequencing a short region of the $r p o B$ gene, we have determined that $K$. variicola and K. pseudoneumoniae isolates could be misidentified as K. pneumoniae by routine biochemical methods and that this biochemical misidentification is especially relevant within the K. oxytoca complex, where most isolates identified as $K$. oxytoca belong to K. michiganensis and, to a lesser extent, to K. grimontii and Raoultella species. Attending to the presence of virulence determinants, the production of siderophores or the ability to form biofilms, we were unable to distinguish between commensal Klebsiella strains isolated from healthy subjects and Klebsiella strains isolated from community-acquired infections or NICU nosocomial outbreaks. The only difference observed was the antibiotic susceptibility profiles since the strains isolated from the NICU's outbreak were ESBLs producers and harboured CTX-M-15 genes. Finally, while this study reveals that the human microbiota could constitute a reservoir of commensal Klebsiella isolates with the potential to become pathogenic, we have also demonstrated that it could be envisaged as a potential source of novel antimicrobials to combat the increasing threat of antibiotic resistance in Klebsiella spp.

Supplementary Materials: The following are available online at https:/ / www.mdpi.com/article/10 .3390 / microorganisms9112344/s1, Figure S1: PFGE profiles of the isolates from the K. pneumoniae (A) and K. oxytoca complex (B), Figure S2: Plasmid profiles of isolates from K. pneumoniae (A) and K. oxytoca complex, Table S1: Oligonucleotides used in this study.

Author Contributions: Conceptualization, J.M.R. and A.M.-B.; methodology, M.G., A.V. and R.d.C.; software, R.d.C. and A.M.-B.; validation, M.G., A.V., J.M.R., R.d.C. and A.M.-B.; formal analysis, R.d.C., J.M.R. and A.M.-B.; investigation, M.G., A.V., R.d.C., J.M.R. and A.M.-B.; resources, R.d.C. and J.M.R.; data curation, A.M.-B.; writing-original draft preparation, A.M.-B.; writing-review and editing, M.G., A.V., R.d.C. and J.M.R.; supervision, J.M.R. and A.M.-B.; project administration, 
J.M.R.; funding acquisition, J.M.R. All authors have read and agreed to the published version of the manuscript.

Funding: This research was funded by Ministerio de Ciencia e Innovación (Spain), grant numbers CSD2007-00063 (FUN-C-FOOD, Consolider-Ingenio 2010), AGL2010-15420 and PID2019-105606RB-I00.

Institutional Review Board Statement: The study was conducted according to the guidelines of the Declaration of Helsinki, and approved by the Ethical Committee of Clinical Research of the Hospital Clínico San Carlos (Madrid, Spain) (protocol code 10/017-E).

Informed Consent Statement: Informed consent was obtained from all subjects involved in the study.

Data Availability Statement: The rpoB gene sequences obtained in this study were deposited in the GenBank database, under the accession numbers KJ499842 to KJ499903.

Acknowledgments: We would like to express our gratitude to Fernando Cháves, the research group UCM 920080 and the departments of Microbiology of the hospitals Doce de Octubre and Ramón y Cajal (Madrid, Spain) for providing us with the Klebsiella isolates used in this work.

Conflicts of Interest: The authors declare no conflict of interest. The funders had no role in the design of the study; in the collection, analyses, or interpretation of data; in the writing of the manuscript, or in the decision to publish the results.

\section{References}

1. Podschun, R.; Ullmann, U. Klebsiella spp. as nosocomial pathogens: Epidemiology, taxonomy, typing methods, and pathogenicity factors. Clin. Microbiol. Rev. 1998, 11, 589-603. [CrossRef] [PubMed]

2. Bengoechea, J.A.; Sa Pessoa, J. Klebsiella pneumoniae infection biology: Living to counteract host defences. FEMS Microbiol. Rev. 2019, 43, 123-144. [CrossRef]

3. Blin, C.; Passet, V.; Touchon, M.; Rocha, E.P.C.; Brisse, S. Metabolic diversity of the emerging pathogenic lineages of Klebsiella pneumoniae. Environ. Microbiol. 2017, 19, 1881-1898. [CrossRef]

4. Brisse, S.; Verhoef, J. Phylogenetic diversity of Klebsiella pneumoniae and Klebsiella oxytoca clinical isolates revealed by randomly amplified polymorphic DNA, gyrA and parC genes sequencing and automated ribotyping. Int. J. Syst. Evol. Microbiol. 2001, 51, 915-924. [CrossRef]

5. Rodrigues, C.; Passet, V.; Rakotondrasoa, A.; Diallo, T.A.; Criscuolo, A.; Brisse, S. Description of Klebsiella africanensis sp. nov., Klebsiella variicola subsp. tropicalensis subsp. nov. and Klebsiella variicola subsp. variicola subsp. nov. Res. Microbiol. 2019, 170, 165-170. [CrossRef]

6. Merla, C.; Rodrigues, C.; Passet, V.; Corbella, M.; Thorpe, H.A.; Kallonen, T.V.S.; Zong, Z.; Marone, P.; Bandi, C.; Sassera, D.; et al. Description of Klebsiella spallanzanii sp. nov. and of Klebsiella pasteurii sp. nov. Front. Microbiol. 2019, 10, 2360. [CrossRef]

7. Long, S.W.; Linson, S.E.; Ojeda Saavedra, M.; Cantu, C.; Davis, J.J.; Brettin, T.; Olsen, R.J. Whole-genome sequencing of human clinical Klebsiella pneumoniae isolates reveals misidentification and misunderstandings of Klebsiella pneumoniae, Klebsiella variicola, and Klebsiella quasipneumoniae. mSphere 2017, 2, e00290-17. [CrossRef]

8. Chen, Y.; Brook, T.C.; Soe, C.Z.; O’Neill, I.; Alcon-Giner, C.; Leelastwattanagul, O.; Phillips, S.; Caim, S.; Clarke, P.; Hall, L.J.; et al. Preterm infants harbour diverse Klebsiella populations, including atypical species that encode and produce an array of antimicrobial resistance- and virulence-associated factors. Microb. Genom. 2020, 6, e000377. [CrossRef] [PubMed]

9. Conlan, S.; Kong, H.H.; Segre, J.A. Species-level analysis of DNA sequence data from the NIH Human Microbiome Project. PLoS ONE 2012, 7, e47075. [CrossRef] [PubMed]

10. Amaretti, A.; Righini, L.; Candeliere, F.; Musmeci, E.; Bonvicini, F.; Gentilomi, G.A.; Rossi, M.; Raimondi, S. Antibiotic resistance, virulence factors, phenotyping, and genotyping of non-Escherichia coli Enterobacterales from the gut microbiota of healthy subjects. Int. J. Mol. Sci. 2020, 21, 1847. [CrossRef]

11. Chung, D.R.; Lee, H.; Park, M.H.; Jung, S.I.; Chang, H.H.; Kim, Y.S.; Son, J.S.; Moon, C.; Kwon, K.T.; Ryu, S.Y.; et al. Fecal carriage of serotype K1 Klebsiella pneumoniae ST23 strains closely related to liver abscess isolates in Koreans living in Korea. Eur. J. Clin. Microbiol. Infect. Dis. 2012, 31, 481-486. [CrossRef] [PubMed]

12. Gorrie, C.L.; Mirceta, M.; Wick, R.R.; Edwards, D.J.; Thomson, N.R.; Strugnell, R.A.; Pratt, N.F.; Garlick, J.S.; Watson, K.M.; Pilcher, D.V.; et al. Gastrointestinal carriage is a major reservoir of Klebsiella pneumoniae infection in intensive care patients. Clin. Infect. Dis. 2017, 65, 208-215. [CrossRef] [PubMed]

13. Martin, R.M.; Cao, J.; Brisse, S.; Passet, V.; Wu, W.; Zhao, L.; Malani, P.N.; Rao, K.; Bachman, M.A. Molecular epidemiology of colonizing and infecting isolates of Klebsiella pneumoniae. mSphere 2016, 1, e00261-16. [CrossRef]

14. Clark, R.; Powers, R.; White, R.; Bloom, B.; Sanchez, P.; Benjamin, D.K., Jr. Nosocomial infection in the NICU: A medical complication or unavoidable problem? J. Perinatol. 2004, 24, 382-388. [CrossRef] [PubMed]

15. Fernández, L.; Langa, S.; Martín, V.; Maldonado, A.; Jiménez, E.; Martín, R.; Rodríguez, J.M. The human milk microbiota: Origin and potential roles in health and disease. Pharmacol. Res. 2013, 69, 1-10. [CrossRef] [PubMed] 
16. Rodríguez-Cruz, M.; Alba, C.; Aparicio, M.; Checa, M.Á.; Fernández, L.; Rodríguez, J.M. Effect of sample collection (manual expression vs. pumping) and skimming on the microbial profile of human milk using culture techniques and metataxonomic analysis. Microorganisms 2020, 8, 1278. [CrossRef]

17. Martín, V.; Maldonado, A.; Rodríguez-Baños, M.; del Campo, R.; Rodríguez, J.M.; Jiménez, E. Sharing of bacterial strains between breast milk and infant faeces. J. Hum. Lact. 2012, 28, 36-44. [CrossRef] [PubMed]

18. Thompson, J.D.; Higgins, D.G.; Gibson, T.J. CLUSTAL W: Improving the sensitivity of progressive multiple sequence alignment through sequence weighting, position-specific gap penalties and weight matrix choice. Nucleic Acids Res. 1994, 22, 4673. [CrossRef]

19. Tamura, K.; Stecher, G.; Peterson, D.; Filipski, A.; Kumar, S. MEGA6: Molecular Evolutionary Genetics Analysis version 6.0. Mol. Biol. Evol. 2013, 30, 2725-2729. [CrossRef]

20. Saitou, N.; Nei, M. The neighbor-joining method: A new method for reconstructing phylogenetic trees. Mol. Biol. Evol. 1987, 4, 406-425.

21. Kaufmann, M.E. Pulsed-field gel electrophoresis. In Molecular Bacteriology: Protocols and Clinical Applications; Woodford, N., Johnson, A.P., Eds.; Humana Press: Totowa, NJ, USA, 1998; pp. 33-50.

22. Jarlier, V.; Nicolas, M.-H.; Fournier, G.; Philippon, A. Extended broad-spectrum $\beta$-lactamases conferring transferable resistance to newer $\beta$-lactam agents in Enterobacteriaceae: Hospital prevalence and susceptibility pattern. Rev. Infect. Dis. 1988, 10, 867-878. [CrossRef]

23. Woodford, N.; Fagan, E.J.; Ellington, M.J. Multiplex PCR for rapid detection of genes encoding CTX-M extended-spectrum (beta)-lactamases. J. Antimicrob. Chemother. 2006, 57, 154-155. [CrossRef] [PubMed]

24. Wang, H.; Kelkar, S.; Wu, W.; Chen, M.; Quinn, J.I. Clinical isolates of Enterobacteriaceae producing extended-spectrum beta-lactamases: Prevalence of CTX-M-3 at a hospital in China. Antimicrob. Agents Chemother. 2003, 47, 790-793. [CrossRef] [PubMed]

25. Magiorakos, A.P.; Srinivasan, A.; Carey, R.B.; Carmeli, Y.; Falagas, M.E.; Giske, C.G.; Harbarth, S.; Hindler, J.F.; Kahlmeter, G.; Olsson-Liljequist, B.; et al. Multidrug-resistant, extensively drug-resistant and pandrug-resistant bacteria: An international expert proposal for interim standard definitions for acquired resistance. Clin. Microbiol. Infect. 2012, 18, 268-281. [CrossRef]

26. Fang, C.T.; Chuang, Y.P.; Shun, C.T.; Chang, S.C.; Wang, J.T. A novel virulence gene in Klebsiella pneumoniae strains causing primary liver abscess and septic metastatic complications. J. Exp. Med. 2004, 199, 697-705. [CrossRef]

27. Yu, W.L.; Ko, W.C.; Cheng, K.C.; Lee, H.C.; Ke, D.S.; Lee, C.C.; Fung, C.P.; Chuang, Y.C. Association between rmpA and magA genes and clinical syndromes caused by Klebsiella pneumoniae in Taiwan. Clin. Infect. Dis. 2006, 42, 1351-1358. [CrossRef] [PubMed]

28. Shon, A.S.; Bajwa, R.P.; Russo, T.A. Hypervirulent (hypermucoviscous) Klebsiella pneumoniae: A new and dangerous breed. Virulence 2013, 4, 107-118. [CrossRef] [PubMed]

29. Fletcher, M. Adhesion and biofilm development of acetate-, propionate-, and butyrate-degrading microorganisms on glass surfaces. Can. J. Microbiol. 1977, 23, 1-6. [CrossRef]

30. O'Toole, G.A.; Kolter, R. Initiation of biofilm formation in Pseudomonas fluorescens WCS365 proceeds via multiple, convergent signalling pathways: A genetic analysis. Mol. Microbiol. 1998, 28, 449-461. [CrossRef] [PubMed]

31. Schwyn, B.; Neilands, J.B. Universal chemical assay for the detection and determination of siderophores. Anal. Biochem. 1987, 160, 47-56. [CrossRef]

32. Tagg, J.R.; Dajani, A.S.; Wannamaker, L.W. Bacteriocins of Gram-positive bacteria. Bacteriol. Rev. 1976, 40, 722-756. [CrossRef]

33. Harris, L.J.; Daescheyl, M.A.; Stiles, M.E.; Klaenhammer, T.R. Antimicrobial activity of lactic acid bacteria against Listeria monocytogenes. J. Food Prot. 1999, 52, 384-887. [CrossRef]

34. European Centre for Disease Prevention and Control. Antimicrobial Resistance in the EU/EEA (EARS-Net)—Annual Epidemiological Report 2019; ECDC: Stockholm, Sweden, 2020.

35. Boye, K.; Hansen, D.S. Sequencing of $16 \mathrm{~S}$ rDNA of Klebsiella: Taxonomic relations within the genus and to other Enterobacteriaceae. Int. J. Med. Microbiol. 2003, 292, 495-503. [CrossRef] [PubMed]

36. Drancourt, M.; Bollet, C.; Carta, A.; Rousselier, P. Phylogenetic analyses of Klebsiella species delineate Klebsiella and Raoultella gen. nov., with description of Raoultella ornithinolytica comb. nov., Raoultella terrigena comb. nov. and Raoultella planticola comb. nov. Int. J. Syst. Evol. Microbiol. 2001, 51, 925-932. [CrossRef] [PubMed]

37. Garza-Ramos, U.; Barrios-Camacho, H.; Moreno-Domínguez, S.; Toribio-Jiménez, J.; Jardón-Pineda, D.; Cuevas-Peña, J.; SánchezPérez, A.; Duran-Bedolla, J.; Olguín-Rodriguez, J.; Román-Román, A. Phenotypic and molecular characterization of Klebsiella spp. isolates causing community-acquired infections. New Microbes New Infect. 2018, 23, 17-27. [CrossRef]

38. Hajjar, R.; Ambaraghassi, G.; Sebajang, H.; Schwenter, F.; Su, S.H. Raoultella ornithinolytica: Emergence and resistance. Infect. Drug Resist. 2020, 13, 1091-1104. [CrossRef] [PubMed]

39. Mehmood, H.; Pervin, N.; Israr Ul Haq, M.; Kamal, K.R.; Marwat, A.; Khan, M. A rare case of Raoultella planticola urinary tract infection in a patient with immunoglobulin a nephropathy. J. Investig. Med. High Impact Case Rep. 2018, 6, 2324709618780422.

40. Chapman, P.; Forde, B.M.; Roberts, L.W.; Bergh, H.; Vesey, D.; Jennison, A.V.; Moss, S.; Paterson, D.L.; Beatson, S.A.; Harris, P.N.A. Genomic Investigation reveals contaminated detergent as the source of an extended-spectrum- $\beta$-lactamase-producing Klebsiella michiganensis outbreak in a neonatal unit. J. Clin. Microbiol. 2020, 58, e01980-19. [CrossRef]

41. Rodríguez-Medina, N.; Barrios-Camacho, H.; Duran-Bedolla, J.; Garza-Ramos, U. Klebsiella variicola: An emerging pathogen in humans. Emerg. Microbes Infect. 2019, 8, 973-988. [CrossRef] 
42. Perdigão, J.; Caneiras, C.; Elias, R.; Modesto, A.; Spadar, A.; Phelan, J.; Campino, S.; Clark, T.G.; Costa, E.; Saavedra, M.J.; et al. Genomic epidemiology of carbapenemase producing Klebsiella pneumoniae strains at a northern Portuguese hospital enables the detection of a misidentified Klebsiella variicola KPC-3 producing strain. Microorganisms 2020, 8, 1986. [CrossRef]

43. Nordmann, P.; Cuzon, G.; Naas, T. The real threat of Klebsiella pneumoniae carbapenemase-producing bacteria. Lancet Infect. Dis. 2009, 9, 228-236. [CrossRef]

44. Fiett, J.; Palucha, A.; Miaczynska, B.; Stankiewicz, M.; Przondo-Mordarska, H.; Hryniewicz, W.; Gniadkowski, M. A novel complex mutant beta-lactamase, TEM-68, identified in a Klebsiella pneumoniae isolate from an outbreak of extended-spectrum beta-lactamase-producing Klebsiellae. Antimicrob. Agents Chemother. 2000, 44, 1499-1505. [CrossRef]

45. Bingen, E.H.; Desjardins, P.; Arlet, G.; Bourgeois, F.; Mariani-Kurkdjian, P.; Lambert-Zechovsky, N.Y.; Denamur, E.; Philippon, A.; Elion, J. Molecular epidemiology of plasmid spread among extended broad-spectrum beta-lactamase-producing Klebsiella pneumoniae isolates in a pediatric hospital. J. Clin. Microbiol. 1993, 31, 179-184. [CrossRef] [PubMed]

46. San Millan, A. Evolution of plasmid-mediated antibiotic resistance in the clinical context. Trends Microbiol. 2018, 26, 978-985. [CrossRef] [PubMed]

47. Hennequin, C.; Robin, F. Correlation between antimicrobial resistance and virulence in Klebsiella pneumoniae. Eur. J. Clin. Microbiol. Infect. Dis. 2016, 35, 333-341. [CrossRef]

48. Beceiro, A.; Tomás, M.; Bou, G. Antimicrobial resistance and virulence: A successful or deleterious association in the bacterial world? Clin. Microbiol. Rev. 2013, 26, 185-230. [CrossRef] [PubMed]

49. Brisse, S.; Fevre, C.; Passet, V.; Issenhuth-Jeanjean, S.; Tournebize, R.; Diancourt, L.; Grimont, P. Virulent clones of Klebsiella pneumoniae: Identification and evolutionary scenario based on genomic and phenotypic characterization. PLoS ONE 2009, 4, e4982. [CrossRef]

50. Izquierdo, L.; Coderch, N.; Piqué, N.; Bedini, E.; Corsaro, M.M.; Merino, S.; Fresno, S.; Tomas, J.M.; Regué, M. The Klebsiella pneumoniae wabG gene: Role in biosynthesis of the core lipopolysaccharide and virulence. J. Bacteriol. 2003, 185, 7213-7221. [CrossRef]

51. Regué, M.; Hita, B.; Piqué, N.; Izquierdo, L.; Merino, S.; Fresno, S.; Benedí, V.J.; Tomás, J.M. A gene, uge, is essential for Klebsiella pneumoniae virulence. Infect. Immun. 2004, 72, 54-61. [CrossRef]

52. Fursova, N.K.; Astashkin, E.I.; Ershova, O.N.; Aleksandrova, I.A.; Savin, I.A.; Novikova, T.S.; Fedyukina, G.N.; Kislichkina, A.A.; Fursov, M.V.; Kuzina, E.S.; et al. Multidrug-resistant Klebsiella pneumoniae causing severe infections in the neuro-ICU. Antibiotics 2021, 10, 979. [CrossRef]

53. Ma, L.C.; Fang, C.T.; Lee, C.Z.; Shun, C.T.; Wang, J.T. Genomic heterogeneity in Klebsiella pneumoniae strains is associated with primary pyogenic liver abscess and metastatic infection. J. Infect. Dis. 2005, 192, 117-128. [CrossRef] [PubMed]

54. Dogan, O.; Vatansever, C.; Atac, N.; Albayrak, O.; Karahuseyinoglu, S.; Sahin, O.E.; Kilicoglu, B.K.; Demiray, A.; Ergonul, O.; Gönen, M.; et al. Virulence determinants of colistin-resistant K. pneumoniae high-risk clones. Biology (Basel) $2021,10,436$. [CrossRef] [PubMed]

55. Kramer, J.; Özkaya, Ö.; Kümmerli, R. Bacterial siderophores in community and host interactions. Nat. Rev. Microbiol. 2020, 18, 152-163. [CrossRef] [PubMed]

56. Cotter, P.D.; Hill, C.; Ross, R.P. Bacteriocins: Developing innate immunity for food. Nat. Rev. Microbiol. 2005, 3, 777-788. [CrossRef] [PubMed]

57. Denkovskienė, E.; Paškevičius, Š.; Misiūnas, A.; Stočkūnaitè, B.; Starkevič, U.; Vitkauskienè, A.; Hahn-Löbmann, S.; Schulz, S.; Giritch, A.; Gleba, Y.; et al. Broad and efficient control of Klebsiella pathogens by peptidoglycan-degrading and pore-forming bacteriocins klebicins. Sci. Rep. 2019, 9, 15422. [CrossRef]

58. Martin, R.M.; Bachman, M.A. Colonization, Infection, and the Accessory Genome of Klebsiella pneumoniae. Front. Cell. Infect. Microbiol. 2018, 8, 4. [CrossRef]

59. Ghenea, A.E.; Cioboată, R.; Drocaş, A.I.; Tieranu, E.N.; Vasile, C.M.; Moroşanu, A.; Tieranu, C.G.; Salan, A.-I.; Popescu, M.; Turculeanu, A.; et al. Prevalence and Antimicrobial Resistance of Klebsiella Strains Isolated from a County Hospital in Romania. Antibiotics 2021, 10, 868. [CrossRef]

60. Dorman, M.J.; Short, F.L. Genome watch: Klebsiella pneumoniae: When a colonizer turns bad. Nat. Rev. Microbiol. 2017, 15, 384. [CrossRef]

61. Arroyo, R.; Martín, V.; Maldonado, A.; Jiménez, E.; Fernández, L.; Rodríguez, J.M. Treatment of infectious mastitis during lactation: Antibiotics versus oral administration of Lactobacilli isolated from breast milk. Clin. Infect. Dis. 2010, 50, 1551-1558. [CrossRef]

62. Maldonado, A.; Ruiz-Barba, J.L.; Jiménez-Díaz, R. Purification and genetic characterization of plantaricin NC8, a novel cocultureinducible two-peptide bacteriocin from Lactobacillus plantarum NC8. Appl. Environ. Microbiol. 2003, 69, 383-389. [CrossRef]

63. Bengtsson, T.; Selegård, R.; Musa, A.; Hultenby, K.; Utterström, J.; Sivlér, P.; Skog, M.; Nayeri, F.; Hellmark, B.; Söderquist, B.; et al. Plantaricin NC8 $\alpha \beta$ exerts potent antimicrobial activity against Staphylococcus spp. and enhances the effects of antibiotics. Sci. Rep. 2020, 10, 3580. [CrossRef] [PubMed] 